\title{
Computer-Assisted Detection Of Intracranial Aneurysms Using A Transformer Deep Neural Network In 3D MR Angiography
}

Emily Zhou ( $\square$ ailizhou22@gmail.com )

The Harker School

Felix Huang

Stanford University

\section{Research Article}

Keywords: Transformer, Intracranial Aneurysms, 3D MRA, TOF-MR Angiography, Deep Learning, CNN, Artificial Intelligence, Medical Image Segmentation, Aneurysm Detection, Medical CAD

Posted Date: March 3rd, 2022

DOI: https://doi.org/10.21203/rs.3.rs-1355959/v1

License: (c) This work is licensed under a Creative Commons Attribution 4.0 International License.

Read Full License 


\title{
Computer-Assisted Detection of Intracranial Aneurysms Using a Transformer Deep Neural Network in 3D MR Angiography
}

\author{
Emily Zhou (corresponding) \\ The Harker School \\ 500 Saratoga Avenue, San Jose, CA 95129, USA \\ ailizhou22@gmail.com \\ Felix Huang \\ Stanford University \\ Artificial Intelligence Certificate Program \\ 450 Serra Mall, Stanford, CA 94305, USA \\ fhuang394@gmail.com
}




\begin{abstract}
Intracranial aneurysms, a brain condition caused by weakened blood vessels, result in abnormal bulging or dilation of the intracranial artery walls. Up to $5 \%$ of the global population will suffer from this disease, and early detection is critical. Due to their small size, aneurysms are often missed in initial patient image assessments. Further, the process of interpreting 3D medical images can be time-consuming, error-prone, and require trained radiologists. This study implemented a deep learning model using a 3D Transformer Neural Network to enable the automated computer-assisted detection of aneurysms in 3D TOF-MRA images.

This novel Transformer model demonstrated overall $91.9 \%$ patient-level sensitivity and $95.1 \%$ patient specificity in detecting intracranial aneurysms. Additionally, for aneurysms exceeding $5 \mathrm{~mm}$ in diameter, it reached patient sensitivity of $95.8 \%$ and patient specificity of $96.4 \%$. To our knowledge, this is the first study to implement a Transformer Deep Neural Network for the detection of aneurysms from 3D volumetric TOF-MRA images. The successful results underscore the potential of Transformer models in accelerating diagnoses of cerebrovascular diseases while streamlining radiologists' workflow. This detection method can be applicable for identifying aneurysms in other organs and diseases such as brain tumors.
\end{abstract}

Keywords: Transformer, Intracranial Aneurysms, 3D MRA, TOF-MR Angiography, Deep Learning, CNN, Artificial Intelligence, Medical Image Segmentation, Aneurysm Detection, Medical CAD 


\section{Introduction}

An Intracranial Aneurysm (IA) is a focal abnormal expansion of the wall of an internal cerebral artery. Intracranial aneurysms typically occur at the base of the skull where the artery passes through the subarachnoid space and can compress nearby nerves or tissue. Approximately $3-5 \%$ of the general population develops a brain aneurysm in their lifetime [1]. An estimated 6.5 million people [50], or one in fifty people, in the US have an unruptured brain aneurysm. Even higher incidences of IAs are found in seniors, especially in females and those over seventy-five years of age. With rapidly rising health care costs and increasing shortfalls in Medicare coverage, the early, accurate, and efficient detection of intracranial aneurysms is critical to minimize arterial ruptures or strokes.

However, diagnosis of intracranial aneurysms is challenging because they are usually asymptomatic prior to their rupture. Presently, four major clinical detection methods are used: computerized tomography (CT) or computerized tomography angiography (CTA)], magnetic resonance scanning (MRI/MRA), invasive cerebral angiography, and more invasive lumbar puncture $[2,3,44,45,46]$. Three-dimensional time-of-flight magnetic resonance angiography (3D TOF-MRA) and computed tomography angiography scans (CTAs) are two of the most common imaging techniques for aneurysm diagnosis. Both CTA scans and MRIs can show subtle relationships between aneurysms and important structures around them, including the dynamic growth or shrinkage of aneurysms [47]. However, unlike CT scans or X-rays, MRIs do not use ionizing radiation, nor do they incur the risks of conventional cerebral angiography that requires injection of contrast agents. An extension of MRI technology is magnetic resonance angiography (MRA), which provides greater convenience and safety when diagnosing hemorrhage and ischemic diseases of cerebral arteries and veins. After diagnosis, intracranial aneurysms are treated differently based on their shape and location as well as the patient's age, general condition, and post-onset status, so the accurate characterization of aneurysms is important $[4,48,49]$.

The small size of cerebral aneurysms and the complexities of accurately locating buried blood vessels often lead to cerebral aneurysms being overlooked during patient initial assessments. Recently, the use of Computer-Assisted Detection (CAD) algorithms for the interpretation of medical images has gained popularity as it could increase diagnostic efficiency and accuracy. Traditionally, CAD systems are based on image analysis provided by technicians and neuroradiologists, e.g. threshold-based approaches, probabilistic atlas, vessel curvatures, or graph-cut methods [5-8]. However, this approach relies largely on pre-processing and human expertise, potentially limiting its broader clinical adoption.

A solution that is less vulnerable to subjective human evaluation is the use of algorithms trained with machine learning. Increases in graphical computing power and greater accessibility of MRA and CTA images have led to advances in machine learning techniques for disease detection using medical images. Convolutional Neural Networks 
(CNNs) are a deep learning model that specializes in image processing and have been successfully used in the diagnosis of lung cancer

[10] and diabetic retinopathy [11]. CNNs have also been used to detect intracranial aneurysms, and have performed successfully when combined with deep learning and MRAs [12-14] or CTAs [15,16]. These tools have demonstrated solid results in medical image segmentation and exhibit the potential to match or even exceed the diagnostic accuracy of medical professionals.

However, not only are current CNN approaches limited to variable-sized images but they also work only on predetermined targeted regions rather than entire images, and thus are unable to automatically detect aneurysms in raw MRA \& CT scans [12]. In addition, for CNN models to automatically separate lesions from other anatomical structures, they require high spatial resolution to capture granular levels of segmentation masks. Unfortunately, CNN models typically sacrifice these fine granular features in favor of a wider breadth of contextual information. To address this issue, a unique type of CNN model called UNet was introduced [40]. A typical UNet architecture consists of an encoder and decoder which down-samples for coarse contextual features and then progressively up-samples to fuse them with fine-grained local visual features. Although UNet showed promising performance in medical image segmentation tasks, impacts from distant pixels quickly decay as more convolutional layers are added to the model, which results in UNet models potentially missing the greater context of the disease in the image. Since UNet and other UNet-like CNN models are often misled by local details and therefore overlook lesions, there is a great need for the application of new model architectures to the problem of image-based aneurysm diagnosis.

Over the past year, advances in Transformer Deep Neural Network models have begun addressing the challenges faced by CNNs, accelerating the field of medical image processing. Transformers have produced excellent results in natural language processing (NLP) applications due to the fact that they were designed to handle long-range dependencies for sequence-to-sequence tasks $[17,18]$. Transformer Neural Networks are especially suitable for long sequence tasks because they incorporate self-attention mechanisms, including neural network layers that aggregate information from the entire input, which enable global computation and perfect memory. Transformer Neural Networks also offer great potential for medical image segmentation tasks as they require the understanding of global contexts as well as fine local features. Transformers are particularly suited for this unique application, since aneurysms can be extremely small relative to a patient's overall brain mass, and they need to be carefully identified by type and characterized in fine detail to determine the appropriate medical or surgical intervention. Although a few studies have been published that use Transformer Neural Network models to perform tasks such as brain tumor segmentation, retinal blood vessel segmentation, and polyp segmentation in colonoscopy images [19-26], no studies to our knowledge have employed a Transformer architecture to detect aneurysms. This study proposes the use of a novel Transformer Deep Learning Neural Network approach to identify intracranial aneurysms in 3D MRA scans. 


\section{Methods \& Materials}

\subsection{Dataset}

In this study, we used the TOF-MRA source images and structural MRIs (T1, T2, FLAIR) from the Aneurysm Detection And segMentation Challenge (ADAM) dataset [27]. The dataset includes 113 diverse patient cases, each containing a TOF-MRA image, a structural magnetic resonance image scan (either T1, T2, or FLAIR), and an aligned image, as seen in Figure 1. Of these images, 93 cases contain one or more untreated, unruptured intracranial aneurysms for a total of 129 aneurysms. Each image has dimensions of $512 \times 512$ with up to 140 slices and a size of $15.6 \mathrm{~GB}$ in .nii file format. All images were preprocessed using N4BiasFieldCorrection [42] to correct bias field inhomogeneities. The aneurysm sizes range from 1.0 to $17 \mathrm{~mm}$ in maximum diameter as shown in Table 1.

Table 1. Aneurysms by size in the ADAM dataset.

\begin{tabular}{|c|c|}
\hline Aneurysm Size & \# of Aneurysms \\
\hline$<3 \mathrm{~mm}$ & 144 \\
\hline $3 \mathrm{~mm}-5 \mathrm{~mm}$ & 108 \\
\hline$>5 \mathrm{~mm}$ & 123 \\
\hline
\end{tabular}

Note. 60 scans contain no aneurysms.

For the cases with aneurysms, a mask image provides a manual reference standard. MRAs were interpreted by an interventional neuroradiologist with more than ten years of field experience. The neuroradiologist trained a second rater with extensive medical image analysis experience and familiarity with the annotation software. Upon evaluating the images independently, the first and second raters assessed the full dataset together and reached a final consensus on aneurysm location and size, making any necessary modifications. Their final consensus annotation was used to produce the binary masks and official ground truth data set.

The second dataset used in this research was the BraTS 3D Brain Tumor data set [28-30], which included multimodal scans containing native (T1), post-contrast T1-weighted (T1Gd), T2-weighted (T2), and T2 Fluid Attenuated Inversion Recovery (T2-FLAIR) volumes, as seen in Figure 2. The scans were captured with multiple clinical protocols using scanners from nineteen different institutions. Four raters manually segmented the images using the same annotation protocol approved by trained neuroradiologists. Every volume had $155 \mathrm{MRI}$ image slices. The dataset included 259 High Grade Gliomas (HGG) and 76 Low Grade Gliomas (LGG), for a total of 335 gliomas and a size of $7.96 \mathrm{~GB}$ in .nii file format. 

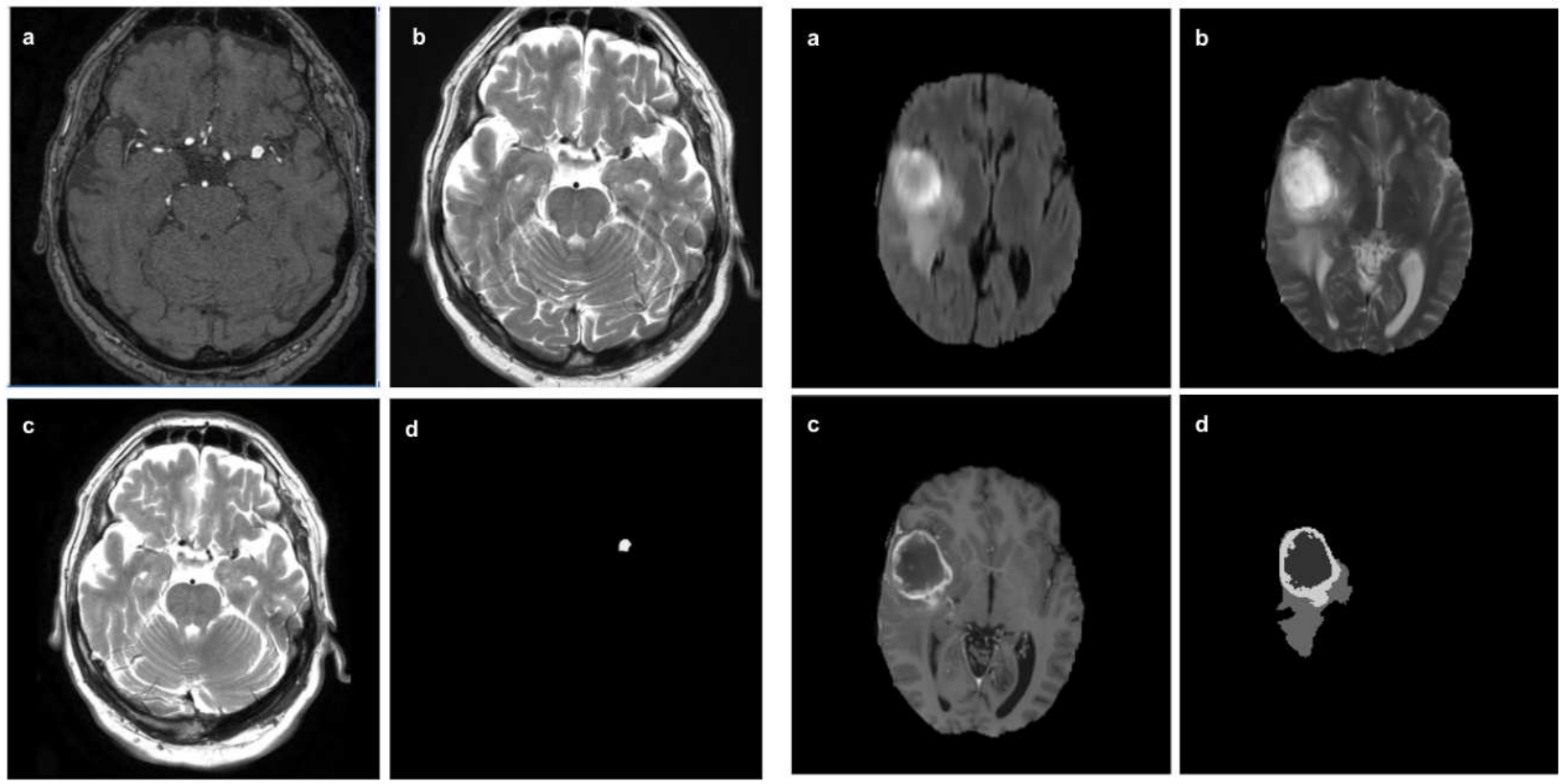

Figure 1. MRA image of the brain with a cerebral aneurysm from the ADAM Dataset. (a) TOF image, (b) structural image, (c) aligned image, and (d) ground truth of mask.

Figure 2. Brain tumor scan from the BraTS 3D

Brain Tumor Dataset. (a) FLAIR shows the whole tumor (WT). The WT displays the full extent of the lesion. (b) T2 shows the Tumor Core (TC). The TC displays the bulk of the tumor, which is typically resected. (c) T1 core-enhanced shows core enhanced tumor structure. (d) Segmentation mask.

\subsection{Methods}

This study utilized anonymized data and was performed in accordance with the relevant guidelines and regulations of the Declaration of Helsinki.

In this study, a Transformer Deep Learning Neural Network model, intrAsegTran, was applied for the segmentation of intracranial aneurysms from 3D MRA images (volume MRA) to determine the presence and location of aneurysms (Figure 3). A Transformer is a neural network consisting of an encoder that maps and retains inputs in a continuous representation with all prior learning and a decoder that simultaneously takes this representation and its own step-by-step outputs to create a final single output; it uses a unique self-attention mechanism to associate different locations in a sequence. This work is based on Segtran [20], which includes a CNN backbone to extract image features, an input/output feature pyramid for upsampling, learnable sinusoidal positional encoding, a squeeze \& expansion transformer layer to contextualize features, and a segmentation head. The model has been successful in tasks similar to aneurysm detection, such as the detection of brain tumors in 3D MRA images. In addition, to handle class imbalance and improve training stability and detection performance, a 
targeted sampling algorithm was adopted and loss-function class weight was tilted to prioritize positive classes. The targeted sampling algorithm produces image patches based on predefined weights. A combined loss function with cross-entropy (CE), binary cross-entropy (BCE), and Dice were deployed. Softmax was used to support potential multi-function classification.

A total of 75 MRA scans of 3D TOF images were used; additionally, a 3D MRA scan, structural image, aligned image, and mask were provided for each image and used for training. The segmentation involved two classes: aneurysms and background. Individual aneurysms are considered 3D-connected components. A bounding box is defined as an imaginary rectangle around the 3D-connected components serving as a reference point for object detection. The Python library connected-components-3d [31] was used to determine the mask bounding box. Data augmentation techniques [32] were also leveraged by randomly applying image modification operations such as horizontal and vertical flipping, shearing, zooming, and rotation to the MRA images. These linear and nonlinear transformations enhanced the training dataset without distorting the images. A $128 \times 144 \times 80$ image patch was produced from the input volume to fit into intrAsegTran. The patch size has minimal impact on the detection accuracy but is not significant. The $128 \times 144 \times 80$ patch is not the largest that can fit into the GPU memory. The patch size was a heuristic choice based on trial and error experimentation; it was selected because it represented the optimum trade-off between GPU memory capacity and the effectiveness of training in terms of granularity and context capturing.

During training, 3D patches of this size were sampled from the entire MRA volume. The image patches were generated in a weighted targeted approach and "overlapping" was allowed. To balance the number of training samples with and without aneurysms, the sampled patches had an $80 \%$ probability of containing aneurysms. In each epoch, approximately 60,000 patches were used to train the model. TOF and Structure were then used for training. Before reaching the network, inputs were normalized to $[-1,1]$. The optimizer used in this model was the adaptive Adam algorithm as it best suited the overall task at hand [44].

Two levels of metrics were used in this research: lesion and patient. Lesion metrics were measured at the aneurysm level and patient metrics were measured at the population level.

Dice Similarity Coefficient (DSC) [33], $\frac{2\left|S{ }_{g}^{1} \cap S{ }_{t}^{1}\right|}{\left|S{ }_{g}^{1}\right|+\left|S{ }_{t}^{1}\right|}$, is used to calculate overlap-based metrics between the ground truth mask and the model-predicted mask. Three different loss functions [34] were used to optimize a weighted sum of Binary Cross-Entropy loss (BCE), Cross-Entropy loss (CE), and Dice loss. Cross-Entropy (CE), $-\sum_{i}^{C} t_{i} \cdot \log \left(s_{i}\right)$, commonly used in machine learning, calculates the difference between two probability distributions. Binary Cross-Entropy (BCE), 
$-\frac{1}{N} \sum_{i=0}^{N} y_{i} \cdot \log \left(\widehat{y_{i}}\right)+\left(1-y_{i}\right) \cdot \log \left(1-\widehat{y_{i}}\right) \quad$, measures the difference between

two probability distributions for a given random variable or set of events; it is frequently

used in classification objectives. Dice loss, $\frac{2 \cdot \sum_{i}^{N} p_{i} g_{i}}{\sum_{i}^{N} p_{i}^{2}+\sum_{i}^{N} g_{i}^{2}}$, was first developed in the 1940s

to calculate the similarity between two images and has been applied in the field of 3D medical image segmentation since 2016.

Four additional models were also evaluated on the same dataset for performance comparisons. The first was DAResUNet [35], an end-to-end 3D CNN segmentation model with an encoder-decoder architecture. DAResUNet also adopted residual blocks and a dual attention block to improve performance. The second model, a 3D-UNet [36] implemented in PyTorch, was top-ranked in the Aneurysm Detection And segMentation Challenge. In this model, the Adam optimizer was applied with the learning rate initialized as $1 \mathrm{E}-4$ and decayed by the cosine curve; a linear combination of Dice loss and Binary Cross-Entropy loss was used as training supervision. The third model was nnU-Net, an extension of the nnU-Net13 [37] with two sampling strategies. The last model evaluated was DCNN with Bag of Tricks [38], an ensemble of five models and the winning entry in the BraTS 2019 challenge.

All training was conducted on AWS p3.8xlarge instances with four NVIDIA V100 Tensor Core GPUs, each with 64GB of memory. A total of $120 \mathrm{k}$ iterations (1,200 epochs) with a batch size of 1 were performed on the model. To save GPU RAM, 3D tasks used only one transform layer.

In the model testing stage, the segmentation prediction of the whole volume was generated by merging the prediction of uniformly sampled patches that could overlap. For each voxel, the highest probability from all enclosing patches was used as its final prediction. The maximum connectivity area was calculated from the final region of vessels then compared against the ground truth mask to determine the degree of overlap. 


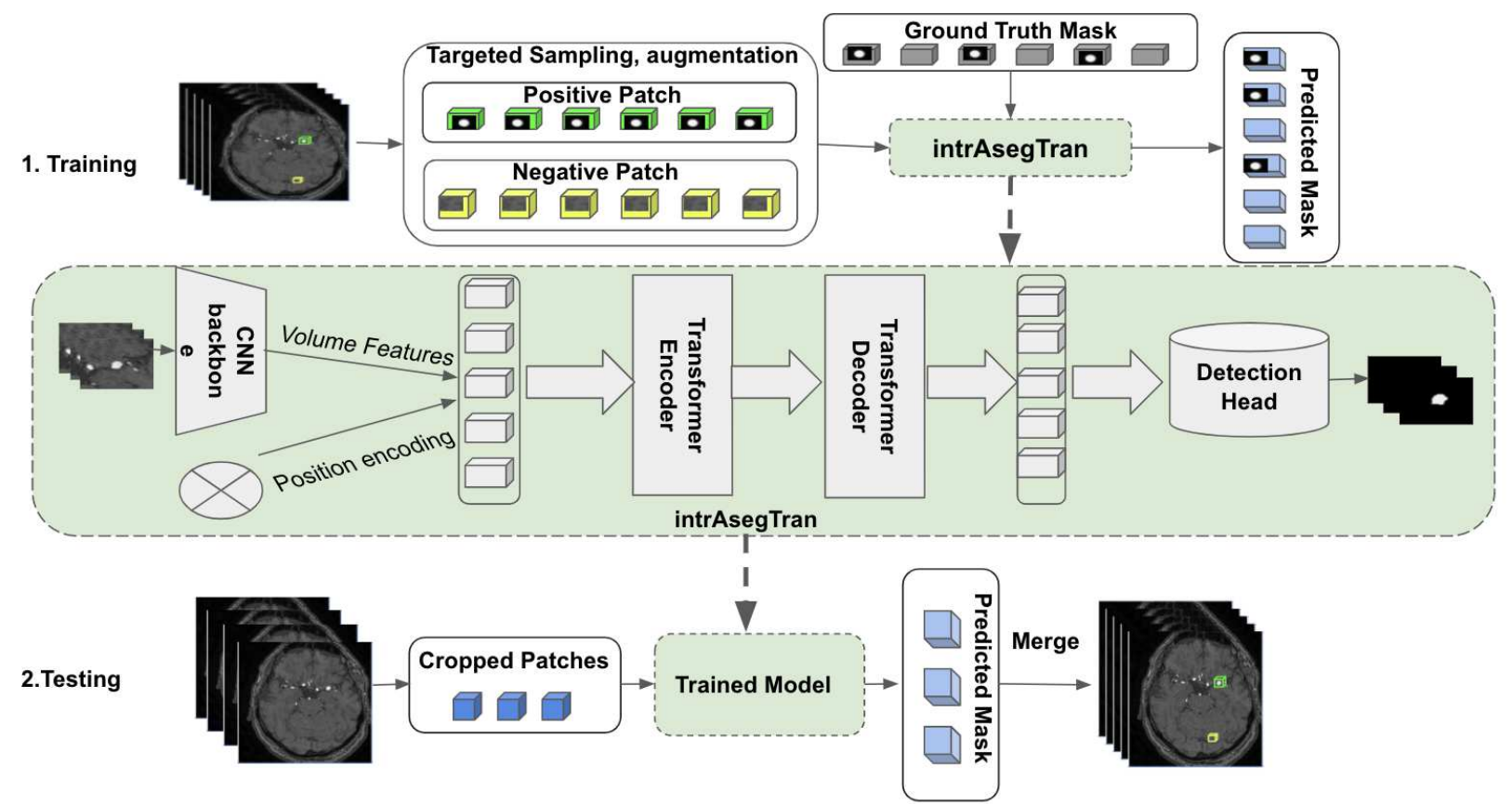

Figure 3. The architecture of the Transformer Deep Learning model.

(1) In the training stage, data were augmented and targeted-sampled. A CNN backbone was used to extract image features, combined with a positional encoding, and fed into the Transformer model. (2) In the testing phase, images were cropped to patches and fed into the trained model, eventually merging together to reconstruct the predicted mask.

\section{Results}

The intrASegTran model performed well and achieved $91.9 \%$ patient sensitivity, $95.1 \%$ patient specificity, $91.1 \%$ lesion precision, and $87.8 \%$ lesion recall (Table 2 ). The model also generated 0.10 false positives per case and a 0.58 dice similarity coefficient score. The subgroup analysis based on lesion size displayed an even better performance on aneurysms larger than $5 \mathrm{~mm}$ (95.8\% patient accuracy) and satisfactory results $(90.35 \%$ patient accuracy) on smaller aneurysms. As illustrated, the intrASegTran model detected a bounding box that successfully overlapped with an actual lesion in a true positive case and made a false negative prediction on a small aneurysm in a second case (Figure 4). 
Table 2. Model performance on aneurysm segmentation.

\begin{tabular}{|c|c|c|c|c|c|c|c|}
\hline Cohort & $\begin{array}{c}\text { Patient } \\
\text { Accuracy } \\
(\%)\end{array}$ & $\begin{array}{c}\text { Patient } \\
\text { Sensitivity } \\
(\%)\end{array}$ & $\begin{array}{c}\text { Patient } \\
\text { Specificity } \\
(\%)\end{array}$ & $\begin{array}{c}\text { Lesion } \\
\text { Precision } \\
(\%)\end{array}$ & $\begin{array}{c}\text { Lesion } \\
\text { Recall } \\
(\%)\end{array}$ & $\begin{array}{c}\text { False Positives } \\
\text { (FP) } \\
\text { per Case }\end{array}$ & DSC \\
\hline Overall & 93.5 & 91.9 & 95.1 & 91.1 & 87.8 & 0.10 & 0.58 \\
\hline$<3 \mathrm{~mm}$ & 90.3 & 84.3 & 96.4 & 91.8 & 82.4 & 0.08 & 0.46 \\
\hline $3-5 \mathrm{~mm}$ & 95.3 & 93.1 & 97.5 & 95.1 & 95.1 & 0.07 & 0.56 \\
\hline$>5 \mathrm{~mm}$ & 95.8 & 94.4 & 97.2 & 91.1 & 88.5 & 0.08 & 0.67 \\
\hline
\end{tabular}

The Transformer model (intrAsegTran) performance compared very well with four previous state-of-the-art, high-performing CNN-based models (Table 3). The Transformer model demonstrated superior performance on the ADAM dataset and comparable performance on the BraTS dataset.

To address the small sample sizes of most medical 3D image datasets, transfer-learning [43], pretraining, and refinement techniques were used to increase model performance. Transfer learning is an active area of research in machine learning focused on using knowledge acquired from solving different, yet relevant, problems, often from other domains, to design and train new models to make better predictions for the problem at hand. When insufficient data exists to train a new large or complex model, these pretrained models can provide a solid starting point to expedite training and boost more accurate results. In this study, a pretrained i3d [50] model was used to abstract image features. The i3d is a Convolutional Neural Network model for video classification that was trained on the Kinetics dataset [45]. It uses image classification architectures with filters and pooling kernels inflated into 3D that produce deep, spatiotemporal classifiers.

Performance results of training with and without pretraining using transfer learning were also compared (Table 4). Pretraining with transfer learning delivered a $21.9 \%$ increase in patient accuracy and a $15.7 \%$ increase in sensitivity for aneurysm detection. 

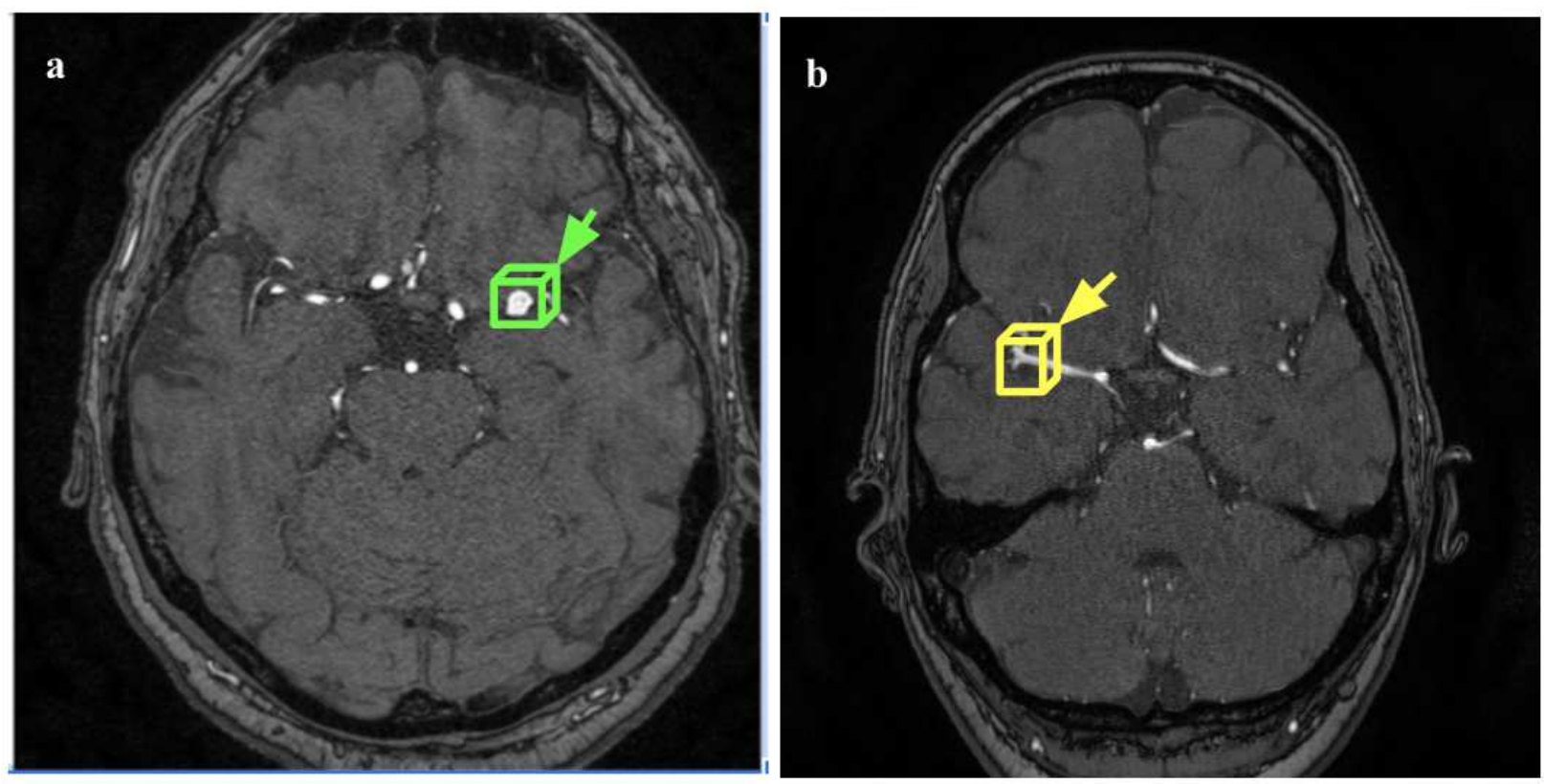

Figure 4. Two intrASegTran model-suggested aneurysms from the test data sets.

In (a) the image illustrates a true-positive model prediction. The model-predicted bounding box (green) is positioned as lower-left-corner coordinates $(346,206,70)$ and upper-right-corner coordinates $(363,224$, 81). Although not shown in Figure 4, the bounding box encircling a white-colored aneurysm is located with lower-left corner coordinates $(343,206,69)$ and upper right corner coordinates $(363,225,81)$. These close coordinates demonstrate that the predicted and actual bounding boxes almost fully overlap. On the other hand, image (b) shows a false-negative prediction (yellow bounding box) with no lesions found due to the aneurysm's unique location and small size of $\sim 1 \mathrm{~mm}$.

Table 3. Model performance comparison on the dataset with aneurysm images (ADAM) and brain tumor images (BraTS 2019).

\begin{tabular}{|c|c|c|c|}
\hline \multicolumn{4}{|c|}{ Aneurysms (ADAM dataset) } \\
\hline Models & Sensitivity (\%) & FPS & DSC \\
\hline ADAM UNet & 68.0 & 0.43 & 0.43 \\
\hline DAResUNet & 86.0 & 0.50 & 0.68 \\
\hline intrAsegTran & 91.1 & 0.10 & 0.58 \\
\hline \multicolumn{4}{|c|}{ Brain Tumors (BraTS 2019 dataset) [20] } \\
\hline Models & ET DSC & WT DSC & TC DSC \\
\hline nnU-Net & 0.737 & 0.894 & 0.807 \\
\hline $\begin{array}{c}\text { DCNN with Bag } \\
\text { of Tricks }\end{array}$ & 0.729 & 0.904 & 0.802 \\
\hline intrAsegTran & 0.740 & 0.895 & 0.817 \\
\hline
\end{tabular}

Note. The upper half of the table shows aneurysm segmentation performance compared with two models: ADAM UNet, the top-performing model in the ADAM Challenge, and DAResUNet, a UNet with dual-attention and residual network. The bottom half of the table shows two brain tumor segmentation models: nnU-Net and DCNN. 
Table 4. Performance improvements of training with transfer learning compared to training without transfer learning.

\begin{tabular}{|c|c|c|c|c|c|c|c|c|}
\hline Model & Cohort & $\begin{array}{c}\text { Patient } \\
\text { Accuracy }\end{array}$ & $\begin{array}{c}\text { Patient } \\
\text { Sensitivity } \\
(\%)\end{array}$ & $\begin{array}{c}\text { Patient } \\
\text { Specificity } \\
(\%)\end{array}$ & $\begin{array}{c}\text { Lesion } \\
\text { Precision } \\
(\%)\end{array}$ & $\begin{array}{c}\text { Lesion } \\
\text { Recall }\end{array}$ & $\begin{array}{c}\text { FP } \\
\text { Per } \\
\text { Case }\end{array}$ & DSC \\
\hline $\begin{array}{c}\text { Without } \\
\text { Transfer } \\
\text { learning }\end{array}$ & Overall & 71.7 & 76.2 & 67.1 & 65.0 & 70.6 & 0.92 & 0.09 \\
\hline $\begin{array}{c}\text { With } \\
\begin{array}{c}\text { Transfer } \\
\text { learning }\end{array}\end{array}$ & Overall & 93.5 & 91.9 & 95.1 & 91.1 & 87.8 & 0.10 & 0.58 \\
\hline \multirow{2}{*}{$\begin{array}{l}\text { Without } \\
\text { Transfer } \\
\text { learning }\end{array}$} & $3-5 \mathrm{~mm}$ & 57.0 & 26.6 & 87.4 & 65.3 & 62.5 & 0.15 & 0.02 \\
\hline & $>5 \mathrm{~mm}$ & 68.4 & 83.5 & 53.3 & 56.6 & 80.3 & 1.28 & 0.13 \\
\hline \multirow{2}{*}{$\begin{array}{c}\text { With } \\
\text { Transfer } \\
\text { learning }\end{array}$} & $<3 \mathrm{~mm}$ & 90.4 & 84.3 & 96.4 & 91.8 & 82.4 & 0.08 & 0.46 \\
\cline { 2 - 9 }$y$ & $>5 \mathrm{~mm}$ & 95.3 & 93.1 & 97.5 & 95.1 & 95.1 & 0.07 & 0.56 \\
\hline
\end{tabular}

In addition to pretraining, targeted sampling and tilted class weight optimizations were implemented to handle negative or positive class imbalances and increase training stability. Evaluations were made with and without these two optimizations (Table 5). With the optimizations, the model showed improvements across all seven metrics and a $12.6 \%$ increase in patience accuracy.

Table 5. Performance improvements of targeted sampling (optimized vs. unoptimized).

\begin{tabular}{|c|c|c|c|c|c|c|c|c|}
\hline Model & Cohort & $\begin{array}{c}\text { Patient } \\
\text { Accuracy } \\
(\%)\end{array}$ & $\begin{array}{c}\text { Patient } \\
\text { Sensitivity } \\
(\%)\end{array}$ & $\begin{array}{c}\text { Patient } \\
\text { Specificity } \\
(\%)\end{array}$ & $\begin{array}{c}\text { Lesion } \\
\text { Precision } \\
(\%)\end{array}$ & $\begin{array}{c}\text { Lesion } \\
\text { Recall }\end{array}$ & $\begin{array}{c}\text { FP } \\
\text { Per Case }\end{array}$ & DSC \\
\hline Optimized & Overall & 93.5 & 91.9 & 95.1 & 91.1 & 87.8 & 0.10 & 0.58 \\
\hline \multirow{3}{*}{ Unoptimized } & Overall & 80.9 & 84.0 & 77.8 & 74.4 & 73.6 & 0.77 & 0.34 \\
\hline & $<3 \mathrm{~mm}$ & 90.4 & 84.3 & 96.4 & 91.8 & 82.4 & 0.08 & 0.46 \\
\cline { 2 - 10 } & $3-5 \mathrm{~mm}$ & 95.3 & 93.1 & 97.5 & 95.1 & 95.1 & 0.07 & 0.56 \\
\cline { 2 - 10 } Optimized & $>5 \mathrm{~mm}$ & 95.8 & 94.4 & 97.2 & 91.1 & 88.5 & 0.08 & 0.67 \\
\hline & $<3 \mathrm{~mm}$ & 77.5 & 70.6 & 84.4 & 71.2 & 66.9 & 0.47 & 0.20 \\
\cline { 2 - 9 } & $3-5 \mathrm{~mm}$ & 82.2 & 75.9 & 88.5 & 78.0 & 71.2 & 0.27 & 0.31 \\
\cline { 2 - 9 } & $>5 \mathrm{~mm}$ & 84.5 & 89.0 & 80.0 & 71.5 & 83.9 & 0.45 & 0.45 \\
\hline
\end{tabular}

\section{Discussion}

The benefits of using CAD to detect intracranial aneurysms have been documented [5-8], and further performance enhancements may result in improved, faster, and more reliable diagnoses. Superior CAD systems with higher sensitivities and lower false positive rates should continue to lower the number of missed aneurysms, as well as 
save time, money, and stress for physicians and patients. Recent medical image processing has led to improved modalities with MRAs providing 3D volumetric data. However, much of the Al medical image research is based on 2D images or 2D architecture with slices of 3D images. Using combined slices of 2D images to represent 3D objects for network inputs reduces accuracy, decreases reliability, introduces sensitivity, and increases the risk of errors.

Although 3D CNNs such as 3D UNet strive to address these issues, relative to the Transformer model, these CNNs not only lose global context when network layers become too deep but also carry a high computational cost.

In this study, we adopted and applied a 3D Transformer Deep Learning model on 3D images for aneurysm detection. We leveraged various data augmentation techniques to improve training and used transfer learning, pretraining, and refinement techniques to increase overall detection performance. With the Transformer model's unique encoding-decoding and self-attention mechanisms, the model can quickly and reliably detect intracranial cerebral aneurysms by processing a scan in 30 seconds on AWS p3.8xlarge instance. The model possessed a sensitivity rate of $91.1 \%$ with less than 0.10 false positives per case- higher performance than those of clinical radiologists who generally detect $60-79 \%$ aneurysms with $0.23-0.37$ false positives per case [39]. Although comparisons with other studies are difficult due to the use of different datasets and evaluation criteria, these results are comparable to or better than those of recent studies [35-38].

The main limitation of this study is the relatively small size of the dataset, which introduces the possibility of sampling bias. In addition, we were limited to conducting a retrospective analysis, as we lacked direct clinical access. Future work should include testing the model performance on larger data sets from different institutions with various imaging equipment and applying the approach to verify performance in real-life clinical diagnostic scenarios. Future work should also focus on adapting this method to detecting life-threatening aneurysms found in other anatomical locations, such as aortic aneurysms, abdominal or thoracic aortic aneurysms, and peripheral aneurysms. Lastly, this 3D Transformer model may be applicable to other diseases with similar lesion-detection profiles in 3D MRA images.

\section{Conclusion}

A Transformer Deep Learning Neural Network with 3D MRA images was applied to aneurysm diagnosis for the first time. The model achieved $91.1 \%$ patient sensitivity and 0.10 false positives per case. It leverages unlimited receptive fields of Transformers to contextualize features and handle both the global picture and fine details for improved segmentation performance. Data preprocessing demonstrated notable efficacy in improving performance, and a targeted sampling strategy was implemented to handle class imbalances. These efforts made the model more robust, boosting overall performance and increasing patient accuracy by $21.9 \%$. In addition, this study indicates that model pretraining and refinement can produce improvements of $12.6 \%$. This CAD tool can complement and accelerate the workflow of neuroradiologists by providing 
more reliable and accurate diagnoses of intracranial aneurysms, resulting in more prompt treatment and improved prognoses while expanding patient access to therapy. These findings could also have an impact on other disease diagnoses, such as detecting brain tumors, that utilize 3D medical angiography images. 


\section{Abbreviations}

IA: Intracranial Aneurysm

3D: 3-Dimensional

ADAM: Aneurysm Detection And segMentation Challenge

AWS: Amazon Web Services

BCE: Binary Cross-Entropy

BraTS: Brain Tumor Segmentation

CE: Cross-Entropy

CT: Core Tumor

TOF-MRA: Time-of-Flight Magnetic Resonance Angiography

CTA: Computed Tomography Angiography

MRI: Magnetic Resonance Imaging

MRA: Magnetic Resonance Angiography

DSA: Digital Subtraction Angiography

DSC: Dice Similarity Coefficient

ET: Enhanced Tumor

FP: False Positive

FN: False Negative

HGG: High Grade Glioma

LGG: Low Grade Glioma

TC: Tumor Core

WT: Whole Tumor

\section{Availability of data and materials}

ADAM dataset: Please contact Aneurysm Detection and Segmentation Challenge to obtain the data [27]. https://adam.isi.uu.nl/data/

BraTS dataset: The datasets used and/or analyzed during the current study are available on BraTS 2019 challenge site [28-30].

https://www.kaggle.com/aryashah2k/brain-tumor-segmentation-brats-2019

\section{Ethics approval and consent to participate}

ADAM 2020 Dataset: Administrative permission was required to access the raw data and permission was granted by ADAM organizer, Dr. Kimberley Timmins.

BraTS 2019 Dataset: Dataset has no copyright (CCO: Public Domain).

\section{Consent for publication}

Consents have been obtained from all authors.

\section{Competing interests}

The authors declare that they have no competing interests. 


\section{Funding}

Not applicable

\section{Authors' contributions}

EZ: Main contributor

FH: Advisor

\section{Acknowledgments}

We appreciate that the datasets for experimentation were provided by the Aneurysm Detection and Segmentation (ADAM) Challenge and the BraTS 2019 Challenge. We also appreciate the responsiveness of the authors of "Medical Image Segmentation Using Squeeze-and-Expansion Transformers" on their online Q\&A forum [20]. 


\section{References}

1. Asaithambi G, Adil MM, Chaudhry SA, Qureshi Al. Incidences of unruptured intracranial aneurysms and subarachnoid hemorrhage: results of a statewide study. J Vasc Interv Neurol. 2014;7(3):14-17.

2. NHS UK. https://www.nhs.uk/conditions/brain-aneurysm/diagnosis/. Accessed 7 Oct 2020.

3. UI Health. https://hospital.uillinois.edu/primary-and-specialty-care/neurology-and-neurosurgery/neurologicalconditions-we-treat/brain-aneurysm/diagnosis-imaging. Accessed 7 Oct 2020.

4. Johns Hopkins Medicine.

https://www.hopkinsmedicine.org/health/treatment-tests-and-therapies/cardiovascular-aneurysm-t reatment. Accessed 10 Oct 2020.

5. Miki S, Hayashi N, Masutani Y, et al. Computer-Assisted Detection of Cerebral Aneurysms in MR Angiography in a Routine Image-Reading Environment: Effects on Diagnosis by Radiologists.

AJNR Am J Neuroradiol. 2016;37(6):1038-1043. doi:10.3174/ajnr.A4671

6. Lauric A, Miller E, Frisken S, Malek AM. Automated detection of intracranial aneurysms based on parent vessel 3D analysis. Med Image Anal. 2010;14(2):149-159. doi:10.1016/j.media.2009.10.005

7. Arimura H, Li Q, Korogi $\mathrm{Y}$, et al. Automated computerized scheme for detection of unruptured intracranial aneurysms in three-dimensional magnetic resonance angiography. Acad Radiol. 2004;11(10):1093-1104. doi:10.1016/j.acra.2004.07.011

8. Yang X, Blezek DJ, Cheng LT, Ryan WJ, Kallmes DF, Erickson BJ. Computer-aided detection of intracranial aneurysms in MR angiography. J Digit Imaging. 2011;24(1):86-95. doi:10.1007/s10278-009-9254-0

9. LeCun, Y., Bengio, Y. \& Hinton, G. Deep learning. Nature 521, 436-444 (2015). https://doi.org/10.1038/nature14539

10. Ardila D, Kiraly AP, Bharadwaj S, et al. End-to-end lung cancer screening with three-dimensional deep learning on low-dose chest computed tomography [published correction appears in Nat Med. 2019 Aug;25(8):1319]. Nat Med. 2019;25(6):954-961. doi:10.1038/s41591-019-0447-x

11. Abràmoff MD, Lou Y, Erginay A, et al. Improved Automated Detection of Diabetic Retinopathy on a Publicly Available Dataset Through Integration of Deep Learning. Invest Ophthalmol Vis Sci. 2016;57(13):5200-5206. doi:10.1167/iovs.16-19964

12. Stember JN, Chang P, Stember DM, et al. Convolutional Neural Networks for the Detection and Measurement of Cerebral Aneurysms on Magnetic Resonance Angiography. J Digit Imaging. 2019;32(5):808-815. doi:10.1007/s10278-018-0162-z

13. Nakao T, Hanaoka S, Nomura Y, et al. Deep neural network-based computer-assisted detection of cerebral aneurysms in MR angiography. J Magn Reson Imaging. 2018;47(4):948-953. doi:10.1002/jmri.25842

14. Ueda D, Yamamoto A, Nishimori M, Shimono T, Doishita S, Shimazaki A, Katayama Y, Fukumoto S, Choppin A, Shimahara Y (2018) Deep learning for MR angiography: automated detection of cerebral aneurysms. Radiology 290(1):187-194.

15. Park A, Chute $C$, Rajpurkar $P$, et al. Deep Learning-Assisted Diagnosis of Cerebral Aneurysms Using the HeadXNet Model. JAMA Netw Open. 2019;2(6):e195600. Published 2019 Jun 5. doi:10.1001/jamanetworkopen.2019.5600.

16. Lv F, Li Q, Liao J, et al. Detection and Characterization of Intracranial Aneurysms with Dual-Energy Subtraction CTA: Comparison with DSA. Acta Neurochir Suppl. 2011;110(Pt 2):239-245. doi:10.1007/978-3-7091-0356-2_43

17. Devlin, Jacob et al. "BERT: Pre-training of Deep Bidirectional Transformers for Language Understanding." NAACL (2019).

18. Sutskever, I., Vinyals, O., Le, Q.V.: Sequence to sequence learning with neural networks. In: NeurIPS (2014).

19. Zhou, Hong-Yu et al. nnFormer: Interleaved Transformer for Volumetric Segmentation. arXiv:2109.03201.

20. Shaohua, Li et al. Medical Image Segmentation Using Squeeze-and-Expansion Transformers. arXiv:2105.09511. 
21. Jun, Eunji et al. Medical Transformer: Universal Brain Encoder for 3D MRI Analysis. arXiv:2104.13633.

22. Hatamizadeh, Ali et al. UNETR: Transformers for 3D Medical Image Segmentation. arXiv:2103.10504.

23. Yun, Boxiang et al. SpecTr: Spectral Transformer for Hyperspectral Pathology Image Segmentation. arXiv:2103.03604.

24. Xie,Yutong et al. CoTr: Efficiently Bridging CNN and Transformer for 3D Medical Image Segmentation. arXiv:2103.03024.

25. Karimi, Davood et al. Convolution-Free Medical Image Segmentation using Transformers. arXiv:2102.13645.

26. Wang W., Chen C., Ding M., Yu H., Zha S., Li J. (2021) TransBTS: Multimodal Brain Tumor Segmentation Using Transformer. In: de Bruijne M. et al. (eds) Medical Image Computing and Computer Assisted Intervention - MICCAI 2021. MICCAI 2021. Lecture Notes in Computer Science, vol 12901. Springer, Cham. https://doi.org/10.1007/978-3-030-87193-2_11.

27. Timmins KM, van der Schaaf IC, Bennink E, et al. Comparing methods of detecting and segmenting unruptured intracranial aneurysms on TOF-MRAS: The ADAM Challenge. Neuroimage. Published online May 2021:118216. doi:10.1016/j.neuroimage.2021.118216

28. B. H. Menze, A. Jakab, S. Bauer, J. Kalpathy-Cramer, K. Farahani, J. Kirby, et al. "The Multimodal Brain Tumor Image Segmentation Benchmark (BRATS)", IEEE Transactions on Medical Imaging 34(10), 1993-2024 (2015) DOI: 10.1109/TMI.2014.2377694

29. S. Bakas, H. Akbari, A. Sotiras, M. Bilello, M. Rozycki, J.S. Kirby, et al., "Advancing The Cancer Genome Atlas glioma MRI collections with expert segmentation labels and radiomic features", Nature Scientific Data, 4:170117 (2017) DOI: 10.1038/sdata.2017.117

30. S. Bakas, M. Reyes, A. Jakab, S. Bauer, M. Rempfler, A. Crimi, et al., "Identifying the Best Machine Learning Algorithms for Brain Tumor Segmentation, Progression Assessment, and Overall Survival Prediction in the BRATS Challenge", arXiv preprint arXiv:1811.02629 (2018).

31. Seung-lab. https://github.com/seung-lab/connected-components-3d. Accessed 10 Oct 2020.

32. A. Mikołajczyk and M. Grochowski, "Data augmentation for improving deep learning in image classification problem," 2018 International Interdisciplinary PhD Workshop (IIPhDW), 2018, doi: 10.1109/IIPHDW.2018.8388338

33. Taha AA, Hanbury A. Metrics for evaluating 3D medical image segmentation: analysis, selection, and tool. BMC Med Imaging. 2015;15:29. Published 2015 Aug 12. doi:10.1186/s12880-015-0068-x.

34. Hang Zhao, Orazio Gallo, Iuri Frosio, Jan Kautz. Loss Functions for Neural Networks for Image Processing. arXiv:1511.08861.

35. Shi, Z., Miao, C., Schoepf, U.J. et al. A clinically applicable deep-learning model for detecting intracranial aneurysm in computed tomography angiography images. Nat Commun 11, 6090 (2020). https://doi.org/10.1038/s41467-020-19527-w.

36. ADMA, https://adam.isi.uu.nl/wp-content/uploads/2020/12/abc_methoddescription_ADAM_challenge.pdf. Accessed 8 Nov 2020.

37. Feifan Wang, Runzhou Jiang, Liqin Zheng, Chun Meng, and Bharat Biswal. 3d u-net based brain tumor segmentation and survival days prediction. In BrainLes Workshop, MICCAI, 2019.

38. Zhao, Yuan et al. "Bag of Tricks for 3D MRI Brain Tumor Segmentation.” BrainLes@MICCAI (2019).

39. Okahara M, Kiyosue H, Yamashita M, et al. Diagnostic accuracy of magnetic resonance angiography for cerebral aneurysms in correlation with 3D-digital subtraction angiographic images: a study of 133 aneurysms. Stroke. 2002;33(7):1803-1808. doi:10.1161/01.str.0000019510.32145.a9

40. Olaf Ronneberger, Philipp Fischer, Thomas Brox. U-Net: Convolutional Networks for Biomedical Image Segmentation. arXiv:1505.04597.

41. Vaswani, A., Shazeer, N., Parmar, N., Uszkoreit, J., Jones, L., Gomez, A.N., Kaiser, L., Polosukhin, I.: Attention is all you need. NeurlPS (2017).

42. Ubuntu Manual. http://manpages.ubuntu.com/manpages/trusty/man1/N4BiasFieldCorrection.1.html. Accessed 8 Nov 2020. 
43. Weiss, K., Khoshgoftaar, T.M. \& Wang, D. A survey of transfer learning. J Big Data 3, 9 (2016). https://doi.org/10.1186/s40537-016-0043-6

44. Introduction to Imaging, Editor(s): Anne G. Osborn, Kathleen B. Digre, Imaging in Neurology, Elsevier, 2016, Pages 2-7, ISBN 9780323447812,

https://doi.org/10.1016/B978-0-323-44781-2.50008-5. (https://www.sciencedirect.com/science/article/pii/B9780323447812500085)

45. Fabio Settecase, Vitaliy L. Rayz, Chapter 6 - Advanced vascular imaging techniques, Editor(s): Steven W. Hetts, Daniel L. Cooke, Handbook of Clinical Neurology, Elsevier, Volume 176, 2021, Pages 81-105, ISSN 0072-9752, ISBN 9780444640345, https://doi.org/10.1016/B978-0-444-64034-5.00016-X. (https://www.sciencedirect.com/science/article/pii/B978044464034500016X)

46. Yang, Zhen Lu et al. "Small Intracranial Aneurysms: Diagnostic Accuracy of CT Angiography." Radiology vol. 285,3 (2017): 941-952. doi:10.1148/radiol.2017162290

47. Novitzke, Jill. "The basics of brain aneurysms: a guide for patients." Journal of Vascular and Interventional Neurology vol. 1,3 (2008): 89-90.

48. Terasaka, S et al. "Surgical approaches for the treatment of aneurysms on the P2 segment of the posterior cerebral artery." Neurosurgery vol. 47,2 (2000): 359-64; discussion 364-6. doi:10.1097/00006123-200008000-00016

49. Yue, W. "Endovascular Treatment of Unruptured Intracranial Aneurysms." Interventional Neuroradiology, vol. 17, no. 4, Dec. 2011, Pages 420-424, doi:10.1177/159101991101700404

50. Carreira, J, Zisserman, A. Quo vadis, action recognition? a new model and the kinetics dataset. In CVPR, 2017 